\title{
Using 3D Non Rigid FFD-Based Method to Register post mortem 3D Histological Data and in vivo MRI of a Baboon Brain
}

\author{
T. Delzescaux ${ }^{1}$, J. Dauguet ${ }^{1,2}$, F. Condé ${ }^{1,3}$, R. Maroy ${ }^{1}$, and V. Frouin ${ }^{1}$ \\ ${ }^{1}$ Service Hospitalier Frédéric Joliot, CEA, Orsay, France \\ ${ }^{2}$ INRIA, Epidaure Project, Sophia Antipolis, France \\ ${ }^{3}$ CNRS, URA 2210, Orsay, France
}

\begin{abstract}
The aim of this study is to propose a protocol allowing to merge informations acquired in vivo (MRI) and post mortem (histology). Departing from the 3-D histological volume and MRI, we first estimated a rigid transformation. Then, an elastic registration method using Free Form Deformation (FFD) was applied to correct the deformations due to the extraction of the brain from skull and the fixation. The final validation of the registration was based on a visual analysis of the matching of internal structures of the brain and the superimposition of cortical sulci.
\end{abstract}

\section{Introduction}

Registration of data acquired in vivo and post mortem allows to confront informations at both macroscopic and microscopic level. The achievement of this task constitutes a complex problem which requires to account for several types of deformations such as: atrophy of the brain after its extraction and fixation, sectioning of the histological slices, distortions during the staining process. In order to study the effects of the different deformations, we studied the first type of deformation alone. We used photographs of the brain taken during the sectioning step. The study presented concerns the registration of the histological volume obtained after the realignment of the photographs [1] with MRI. To correct for these deformations, a FFD-based method [2] was used.

\section{Materials and Methods}

Photographs of the brain were acquired each $0.72 \mathrm{~mm}$ during the sectioning process in the coronal incidence. The resolution in the plane was $0.1 \mathrm{~mm}$. MRI volume was acquired using a T1 sequence with $0.78 \mathrm{~mm} \times 0.78 \mathrm{~mm} \times 1 \mathrm{~mm}$ resolution. The first step consisted in the reconstruction of the histological volume from the photographs. The histological volume has been automatically registered with the MRI (taken as reference) using a rigid transformation based on mutual information as optimisation criterion. From this initialization, an elastic transformation of the histological volume to MRI was estimated using a FFD model based on the optimization of the mutual information. A pyramidal based approach which consisted in increasing gradually the number of control points was chosen. The visualization of the merged images taking into account the external frontiers of the brain as well as the superimposing of the internal structures was used to evaluate the quality of the final registration. Moreover, the main sulci were extracted in both modalities to evaluate in 3-D the quality of the registration in the cortical regions. 


\section{Results}

Figure 1 presents the results of rigid and elastic registrations. Axial and coronal slices of the histological reconstructed volume in its original high resolution have been superimposed with MRI contours after rigid registration (resp. 1.a and 1.b). The same slices are presented after correction of the deformations using FFD method (resp. 1.c and 1.d). The superimposing 3-D sulci for the left hemisphere are illustrated on figure 2 after rigid (figure 2.a) and after FFD registration (figure 2.b).

a)

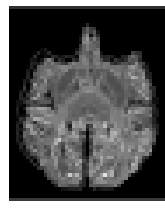

b)

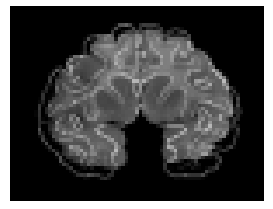

c)

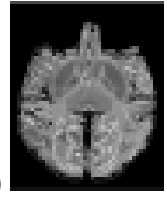

d)

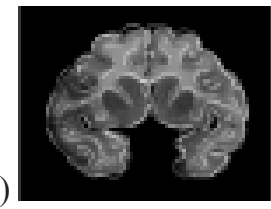

Fig. 1. MRI contours superimposed with histological reconstructed volume in axial and coronal incidence: after rigid registration (resp. a) and b) ), after FFD registration (resp. c) and d) ).

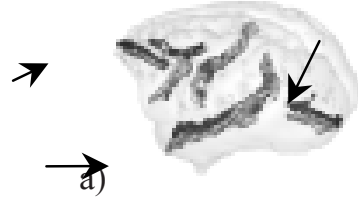

b)

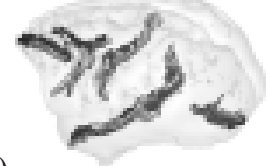

Fig. 2. Light grey and dark grey cortical sulci presented correspond respectively to MRI and histology visualized after rigid registration a) and after FFD registration b).

\section{Discussion}

The preliminary rigid registration provided a correct matching of the internal structures of the brain (basal ganglia). However, important misregistrations were observed particularly in the cortical parts (figure 1.a and 1.b) and required a supplementary elastic registration step. After FFD registration, the main differences of volumes were corrected (figure 1.c and 1.d). In the cortical regions, the FFD registration significantly improved the superimposition of the main sulci extracted in both modalities (figure 2.b).

\section{Conclusion}

The proposed method allowed to register the histological volume with MRI data and to correct efficiently the deformations due to the extraction and fixation of the brain. The evaluation of the registration quality on both internal structures and cortical sulci allowed the validation of the whole process. The first results obtained in this study are promising. Further investigations should be led to take into account the deformations induced by fixation, sectioning and staining of histological slices with respect to other sources of deformations.

\section{References}

1. S. Ourselin, A. Roche, G. Subsol, X. Pennec, and N. Ayache. "Reconstructing a 3-D Structure from Serial Histological Sections". Image and Vision Computing, 19:25-31, 2001.

2. D. Rueckert, L. I. Sonada, C. Hayes, D. L. G. Hill, M. O. Leach, D. J. Hawkes. "Non-rigid Registration Using Free Form Deformations: Application to Breast MR Images", IEEE Transactions on Medical Imaging, Vol. 18, No. 8, pp. 712-722, 1999. 